\title{
AN ANALYTICAL APPROACH TO THE PROBLEM OF MASS LOSS FROM WOLF-RAYET STARS
}

\author{
R. TUROLLA \\ Dept. of Physics, University of Padova \\ Via Marzolo 8 \\ 35131 Padova \\ Italy
}

We investigate the dependence of the wind parameters on the properties of the parent star by means of an approximate analytical approach, largely based upon dimensional arguments (Bandiera and Turolla, 1990). The main assumption we use are

a) WR stars can be treated as homogeneous, pure helium structures;

b) nuclear energy generation is due only to $3-\alpha$ burning;

c) line driving is the mechanism responsible for accelerating the wind;

moreover, all assumptions contained in the original CAK (Castor, Abbott and Klein, 1975) treatment are retained.

Using a simple politropic model for the stellar interior we show that mass loss from WR stars can be characterized just by the stellar mass; it is found that a correlation between mass and mass loss rate of the form $\dot{M} \propto M_{*}^{p}(p \sim 2)$ exists. Such a relation is similar to those ones obtained by Abbott et al. (1986) from observations of WR stars in binary systems and by Turolla, Nobili and Calvani (1988) from the integration of dynamical stellar models.

\section{References}

Abbott, D. C., Bieging, J. H., Churchwell, E. and Torres, A. V. 1986, Astrophys. J., 303, 239

Bandiera, R. and Turolla, R., 1990, Astron. Astrophys., 231, 85

Castor, J. I., Abbott, D. C. and Klein, R. I. 1975, Astrophys. J., 195, 157

Turolla, R., Nobili, L. and Calvani, M. 1988, Astrophys. J., 324, 899 


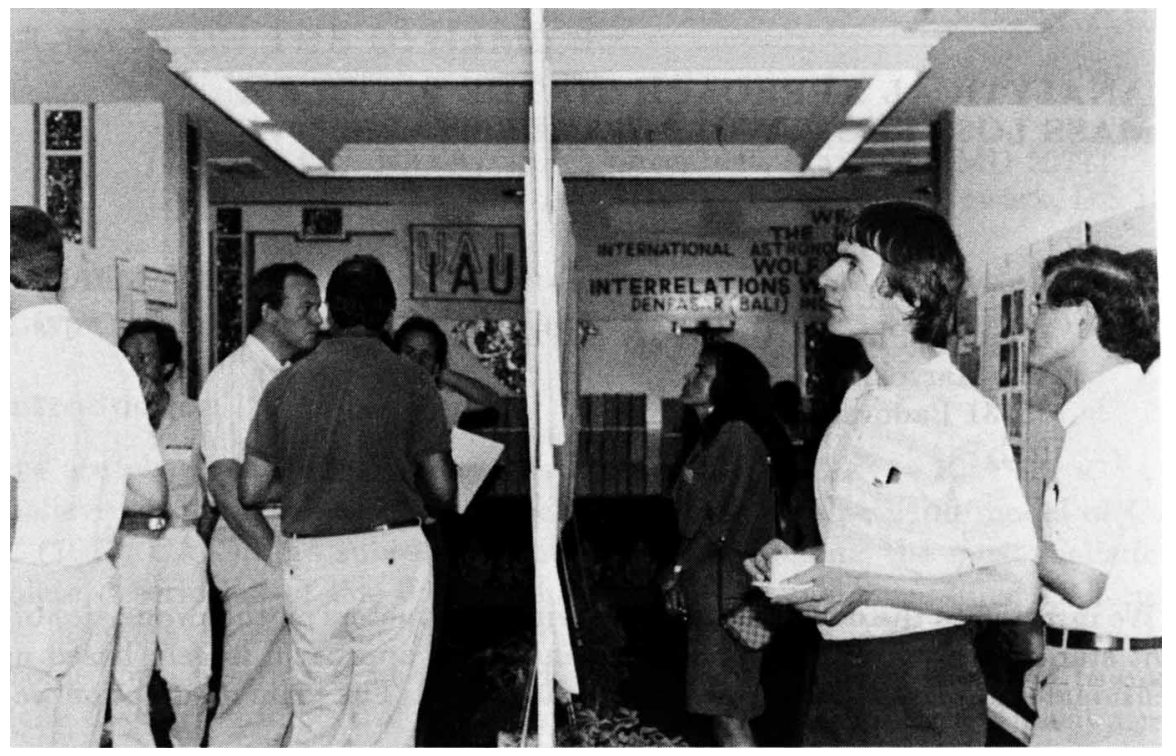

Debray, Matteucci, Lewis, Nomoto 